\title{
Photothermal characterization of plasma surface modifications
}

\author{
J. Pelzl and B.K. Bein \\ Institut für Experimentalphysik, AG Akustik und Optik der Festkörper, \\ Ruhr-Universität Bochum, D-W-4630 Bochum, F.R.G., P.O.Box 102148
}

\begin{abstract}
The basic concept and different detection schemes of the photothermal techniques are briefly described and main applications in plasma surface interactions are reviewed. Results of photoacoustic investigations are presented to demonstrate the depth profiling ability and the lateral resolution of the thermal wave techniques. On metal plates exposed to tokamak divertor plasmas different types of material deposition and annealing effects are identified by frequency dependent measurements. Graphite limiter tiles are found to exhibit thermal depth profiles already before plasma exposure which can be related to the surface roughness and porosity. In a last example frequency variations of the photoacoustic signals obtained from plasma-deposited amorphous hydrogen carbon and deuterium carbon films on silicon are discussed.
\end{abstract}

\section{INTRODUCTION}

In modern plasma-assisted technologies, the plasma and plasma processing are used to generate specific surface properties and structures. With plasma spray techniques heat barrier coatings and protective layers against corrosion can be produced, and plasma vapor deposition and plasma etching of semiconductor materials are used to build up and to pattern the micronic structures of semiconductor devices. In nuclear fusion experiments, on the other hand, plasma wall interactions are not desired but unavoidable: The life time of the wall components of a fusion experiment or reactor is limited by excessive heat loads and plasma-induced erosion and the related too high impurity content in the plasma continues to be one of the major obstacles for the burning plasma.

In both fields we are interested in monitoring and measuring the resulting plasma surface interactions, preferentially with a non-contact remote sensing technique. In plasma processing the product properties, e.g. a plasma-deposited layer thickness, should be controlled in order to adjust the plasma discharge parameters to the desired product, and in nuclear fusion experiments the erosion and redeposition of wall materials should be measured and related to the impurity flux into the plasma, in order to control confinement and burn instabilities.

Photothermal techniques are suited tools to obtain this kind of information. The quantities accessible to a photothermal experiment are the effective thermal properties which are determined by the intrinsic thermal material parameters and by topological effects due to roughness, porosity or artificial structures. Apart from the plasma parameters, in plasma-surface interactions these effective thermal properties of the materials in contact with the plasma are most important. At a given energy flow from the plasma to the walls or substrate the energy dissipation in the solid and its surface temperature are governed by the effective thermal properties. The importance of the surface temperature, e.g. for the erosion of graphite and amorphous carbon films, has been shown by Vietzke et al. [1] in this conference. 


\section{PHOTOTHERMAL METHODS APPLIED TO SOLID STATE MATERIALS}

Photothermal methods are based on thermal wave measurements. The thermal waves can be excited in solids by periodic heating. Excitation and propagation of these waves are governed by the heat diffusion equation and by the strength and localization of the heat sources. Owing to the diffusion process, thermal waves are strongly damped and undergo a phase shift growing with propagation. The penetration depth decreases with increasing modulation frequency of heating. This means, amplitude damping, phase shift and the penetration depth itself can be controlled by the modulation frequency. Since on the other hand the amplitude and phase also depend on the thermal parameters, depthselective information on the thermal effusivity $\epsilon=\sqrt{k \rho c}$ and diffusivity $\alpha=k / \rho c$ can be obtained by measuring the amplitude and phase of thermal waves as a function of the modulation frequency. Here $k, \rho$ and $\mathrm{c}$ are the thermal conductivity, mass density and specific heat capacity, respectively.

For the excitation of thermal waves the absorption of electromagnetic waves, extending from the microwave range over the visible spectrum to X-rays, and particle beams, e.g. electrons and ions are used. Various detection techniques for thermal waves have been developed during the last decade. The most currently used ones are sketched in Fig.1:

- In Photoacoustics the heat released by the sample induces periodical pressure fluctuations in a surrounding gas which are measured by a microphone. - The temperature gradient in the gas layer above the sample can be sensed locally by Laser Beam Deflection of a probe beam passing in grazing incidence near the sample surface. - With the help of Infrared Radiometry the temperature oscillations of the surface are measured as variations of the total emittance. An example is presented in this conference by Gu et al.[2].

- Via the Thermoelastic Effect the periodic heating generates sound waves in the solid which are detected by a piezoelectric transducer. - The temperature oscillations due to thermal waves can also lead to periodical changes of the dielectric and magnetic properties of the material under inspection. These thermal-wave-induced changes can be detected by Thermally Modulated Reflection of light or Thermally Modu-

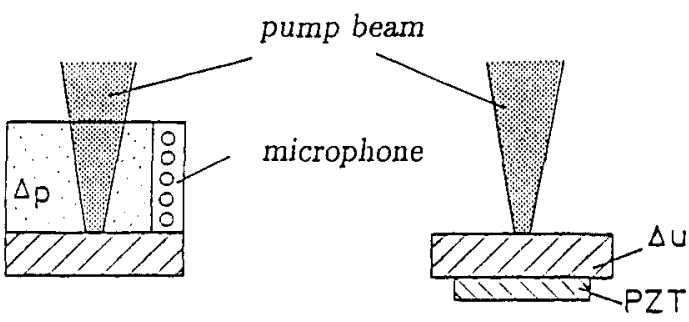

PHOTOACOUSTIC

EFFECT

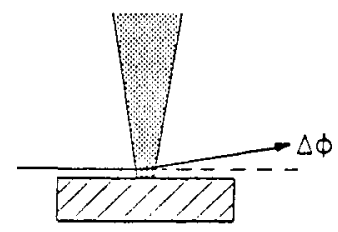

LASER BEAM

DEFLECTION

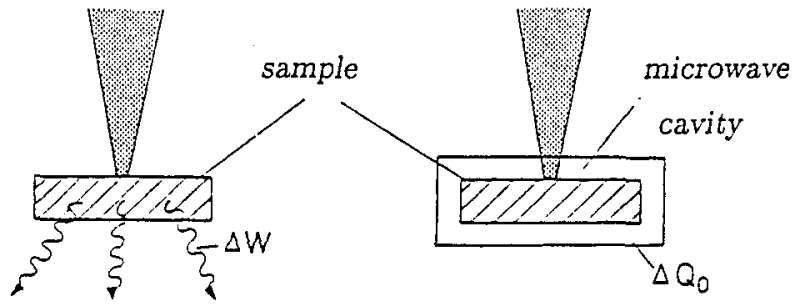

INFRARED

RADIOMETRY
THERMOELASTIC

EFFECT

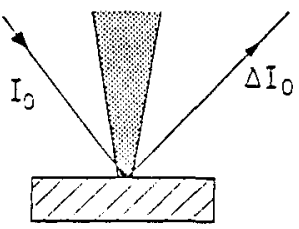

THERMALLY MODULATED

REFLECTION

lated Microwave Resonance.

The amplitude and phase shift with

respect to the exciting modulated laser beam are investigated as a function of the modulation frequency and/or of the position of the heating spot. The detection of the small-amplitude oscillations on a high average signal background or noise level is commonly achieved by the lock-in technique. 


\section{CHARACTERIZATION OF PLASMA SURFACE MODIFICATIONS}

The thermally modulated reflection nowadays is widely used for the evaluation and control in plasma etching of semiconductors [3]. Plasma-sprayed coatings are inspected with respect to coating thickness and delamination by time and frequency dependent infrared radiometry [4]. Apart from material modifications also the energy transfer rate between incident ions and metal samples has become accessible to thermal wave techniques [5]. The examples of photothermal characterization of plasma surface modifications to be reported here refer to the effects of long-time plasma exposure in tokamak devices and to plasma vapor deposition on semiconductor materials. The presented results have been obtained by the photoacoustic technique.

\subsection{Plasma surface modifications on metallic divertor plates}

In the intersection region between the plasma separatrix and a neutralizer plate from the ASDEX divertor, various plasma surface modifications

have been identified by thermal depth profiling. During incorporation in the lower divertor chamber at the outer side, the ion side, the analysed plate had been exposed to approximately 20000 discharges of an average duration of $2.5 \mathrm{~s}$. About 11000 discharges were run only with ohmic heating and 9000 were run with additional neutral beam heating, ICRH and LH heating [6]. The heating regime for the plate and the position of the separatrix intersection also changed considerably, depending on the discharge condi-

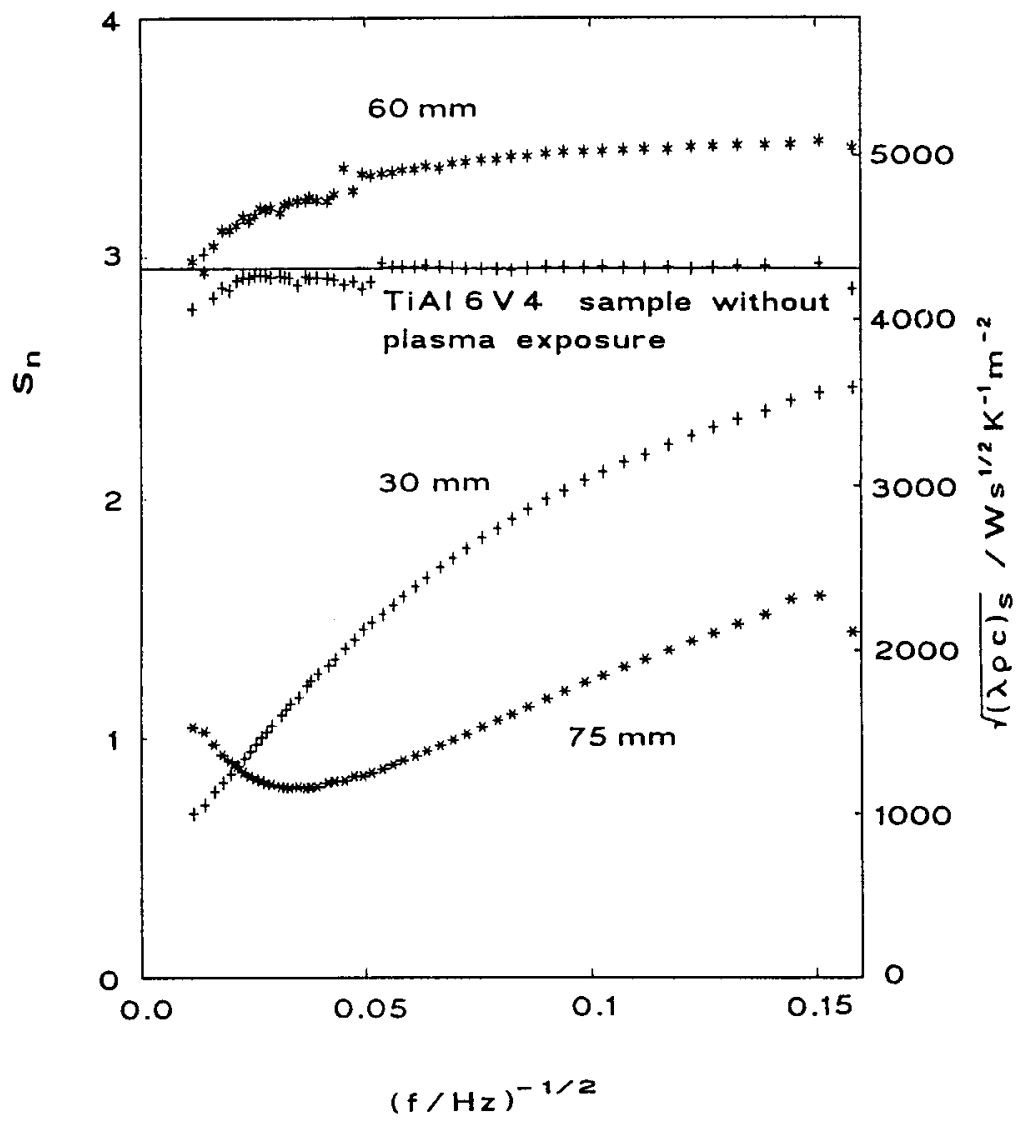

Fig.2: Frequency dependence of the normalized photoacoustic amplitude from different positions on the plasmaexposed divertor plate. tions (plasma current, current of the divertor coils and additional heating). The measurements, presented in Fig.2 refer to the plate material $\left(\mathrm{TiAl}_{6} \mathrm{~V}_{4}\right.$ ) before plasma exposure, to the center of the separatrix intersection (60 $\mathrm{mm}$ from the lower plate edge), to a region of material redeposition in closed multiple layers $(30 \mathrm{~mm})$, and to a region $(75 \mathrm{~mm})$ where cracks of approximately $1 \mu \mathrm{m}$ width are found by SEM. The measurement of the sample without plasma exposure shows an effusivity, which is independent of the penetration depth $\mu \sim f^{-1 / 2}$ and whose value $\epsilon=4210 \mathrm{Ws}^{1 / 2} \mathrm{~m}^{-2} \mathrm{~K}^{-1}$ is in agreement with the literature value. At the center of the separatrix, where material redeposition in layers or droplets can be excluded due to visible light and electron 
micrographs [7], the effusivity value increased by nearly 20 percent. This is related to annealing effects due to thermal cycling at the center of the separatrix. Farther away from the intersection between separatix and neutralizer plate, at position $30 \mathrm{~mm}$, where material redeposition in closed multiple layers of a total thickness of about $10 \mu \mathrm{m}$ has been observed by SEM, the thermal depth profile starts with a very low effusivity value at the surface, which is characteristic for the deposited material, and only for a large penetration depth the original value is reached again. In a limited region above the separatrix $(75 \mathrm{~mm})$ the thermal depth profile shows an inversion as expected for delaminated coatings, where after a thin well conducting first layer a second layer of very low thermal transport properties is found. Electron micrographs of this region show a rough surface with very few thin cracks of about $1 \mu \mathrm{m}$ width. A possible explanation for this delamination is cracking due to short and localized intense heat pulses which were shifted from the normal position of the separatrix into the direction of the plasma torus, as observed by IR thermography in H-regime discharges of ASDEX [8].

\subsection{Plasma surface modifications of graphite limiter plates}

The interpretation of the changes observed for plasma-exposed graphite samples is more complex in comparison to the plasma surface modifications measured on smooth metallic samples. This is due to the fact, that graphites, even before plasma exposure, show a thermal depth profile, a variation of the effusivity value $\epsilon$ with the penetration depth, which is related to the effects of the surface roughness and volume porosity on surface heating processes and heat propagation. The measured depth profiles, both the signal amplitude and the phase shift, can be approximated on the basis of a 3-layer model [9], where the three layers are characterized by their effusivity values and where the first and second layer are characterized by a finite value of the thermal diffusion time $\tau=d^{2} / \alpha$. Here, $\mathrm{d}$ and $\alpha$ represent the geometrical thickness and the thermal diffusivity of the respective layer. For different fine grain graphites (Carbon Lorraine 5890 PT, Ringsdorff EK 98, Schunk \& Ebe FP $151 \mathrm{I})$, the following results have been obtained:

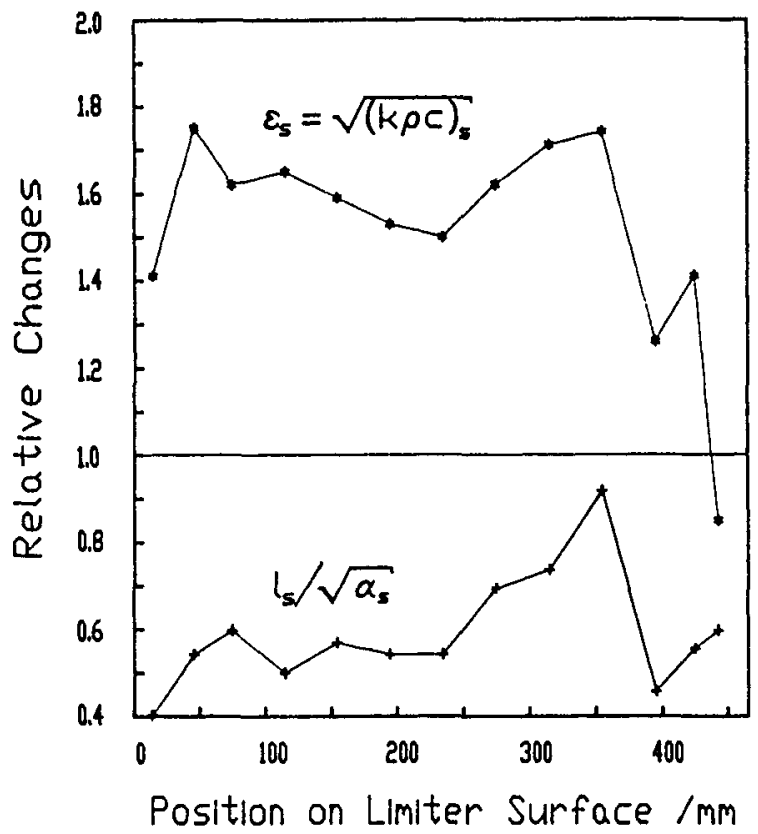

Fig.3: Local changes of the effusivity and thermal diffusion time of the surface layer, measured for a plasma-exposed graphite limiter tile.

1. A very thin surface layer is described by a thermal diffusion time of $20 \mu s<\tau_{s}<40 \mu s$ and by a low effusivity value of $1500 \mathrm{Ws}^{1 / 2} \mathrm{~m}^{-2} \mathrm{~K}^{-1}<\epsilon_{s}<3000 \mathrm{Ws}^{1 / 2} \mathrm{~m}^{-2} \mathrm{~K}^{-1}$;

2. A thicker subsurface layer with thermal diffusion times of $10 \mathrm{~ms}<\tau_{r}=d_{r}^{2} / \alpha_{r}<20 \mathrm{~ms}$ and effusivity values of $8000 \mathrm{Ws}^{1 / 2} \mathrm{~m}^{-2} \mathrm{~K}^{-1}<\epsilon_{r}<12000 \mathrm{Ws}^{1 / 2} \mathrm{~m}^{-2} \mathrm{~K}^{-1}$ is determined by the deep and large open pores and cracks;

3. Deep below the surface, the effusivity is changed by the effects of the porosity on the mass density and thermal conductivity and has values of $6000 \mathrm{Ws}^{1 / 2} \mathrm{~m}^{-2} \mathrm{~K}^{-1}<\epsilon_{p}<8000 \mathrm{Ws} \mathrm{s}^{1 / 2} \mathrm{~m}^{-2} \mathrm{~K}^{-1}$.

On graphite samples exposed to the boundary plasma in the tokamak devices ASDEX and JET, both increased and decreased thermal transport properties have been found, depending on the position of the sample relative to the plasma torus. In regions far away from the plasma which, owing to the magnetic confinement of the charged particles, could only be reached by energetic charge-exchange 
neutrals the effusivity decreased [10], probably due to the induced lattice damage. Increased thermal transport parameters have generally been found in regions close to the plasma torus, regularly heated to higher surface temperatures. The observed changes of the depth profiles have again been interpreted in the frame of the 3-layer model, where the parameters of the first and second layer changed due to plasma surface interactions and where the third layer was assumed to be unchanged. Fig. 3 shows the effusivity $\left(\epsilon_{s}\right)$ and the square root of the thermal diffusion time $\left(\sqrt{\tau_{s}}\right)$ of the surface layer measured as a function of the position on a JET limiter tile.

Quantities normalized with the values of samples without plasma contact are presented in Fig.3.

The changes of the effusivity depth profile observed in the heated regions can be interpreted as modifications of the roughness structure: A densification of the surface material, which is in accordance with a smaller square root value of the thermal diffusion time, $\sqrt{\tau_{s}}=d_{s} / \sqrt{\alpha_{s}}$, and a higher effusivity value of the surface layer $\epsilon_{s}=\sqrt{k_{s}(\rho c)_{s}}$, is accompanied by the formation of larger and deeper open pores and cracks. This can be deduced from the observed increased thermal diffusion time [10] of the second layer, $\sqrt{\tau_{r}}=d_{r} / \sqrt{\alpha_{r}}$, and is in agreement with a statistical geometric-structural model for opaque random surfaces [11], where the large and deep open pores or cracks of an illuminated surface contribute to an increased effective heat capacity. Furthermore, such a formation of vertical surface cracks is supported by observations in heat load experiments run with graphites.

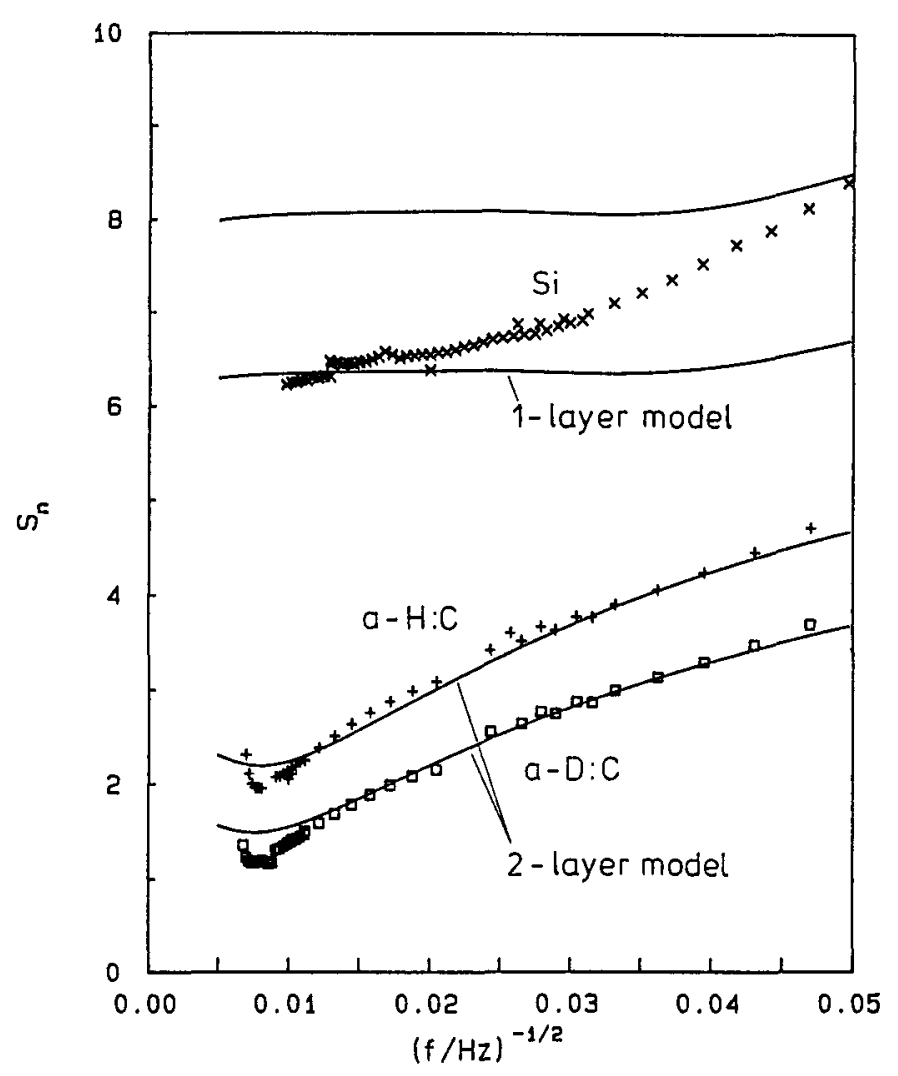

Fig.4: Frequency dependence of the normalized photoacoustic amplitudes from uncoated and plasmacoated Si samples.

\subsection{Amorphous hydrogen carbon films on silicon}

In order to detect and quantify the effects of plasma exposure and plasma deposition of a- $\mathrm{H}: \mathrm{C}$ and aD:C films on silicon, untreated and plasma-exposed samples have been analysed. For the deposition of the films of $1.1 \mu \mathrm{m}$ thickness a $\mathrm{HF}$ discharge was operated at a bias voltage of $450 \mathrm{~V}$ and $3 \mathrm{~Pa}$ methane gas pressure [12]. The normalized amplitudes in Fig.4 show considerably reduced effusivity values at the surface of the plasma-deposited samples, in comparison to the value of the silicon sample. From the phase measurements [13] low thermal diffusivity values of the first layer are derived. This relatively low thermal transport properties can probably be related to lattice damage induced by energetic ion bombardment during the discharge. This is supported by the difference between the samples with the $\mathrm{a}-\mathrm{H}: \mathrm{C}$ and the a-D:C film: The induced lattice damage is larger for the deposition with the heavier deuterium ion. 


\section{CONCLUSIONS}

Thermal wave techniques provide local and depth-dependent thermal properties of surface and subsurface regions extending from a few $\mu m$ to several hundred $\mu m$ below the surface, a range which is hardly accessible to other methods. Photothermal experiments are proved to be suited to analyse plasma surface modifications in controlled fusion devices and in plasma etching and deposition processes. The plasma modifications can be deduced from a thermal wave inspection of the sample before and after plasma exposure as demonstrated by the examples in this contribution. But photothermal methods also offer the possibility for in-situ and remote measurements which can be used to control plasma deposition and etching processes.

\section{Acknowledgements}

This work has been supported by the Max-Planck-Institut für Plasmaphysik, Garching, FRG.

\section{REFERENCES}

1. E. Vietzke, V. Philipps, K. Flaskamp, J. Winter, S. Veprek, P. Koidl, In: Proc. 10th Intern. Symp. on Plasma Chemistry, Bochum, 1991 (Eds. U. Ehlemann, H.G. Lergon, K. Wiesemann), Vol.1, paper 3.1-1.

2. J.H. Gu, B.K. Bein, R. Hüttner, J. Pelzl, D.L. Balageas, A.A. Déom, In: Proc. 10th Intern. Symp. on Plasma Chemistry, Bochum, 1991 (Eds. U. Ehlemann, H.G. Lergon, K. Wiesemann), Vol.3, paper 2.5-6.

3. M. Engelhardt, Solid State Technology, April 1990, 151-156.

4. L.D. Favro, T. Ahmed, D. Crowther, H.J. Jim, P.K. Kuo, R.L. Thomas, X. Wang, SPIE, Vol.1467-Thermosense XIII, 290 (1991).

5. H.F. Winters, H. Coufal, H. Bay, W. Eckstein, In: Proc. 10th Intern. Symp. on Plasma Chemistry, (Eds. U. Ehlemann, H.G. Lergon, K. Wiesemann), 1991, Vol.2, paper 2.2-31.

6. H. Rapp, H. Niedermeyer, M. Kornherr, and the ASDEX team, In: Proc. 14th Symposium on Fusion Technology, Sept. 1986, Avignon, France.

7. B.K. Bein, M. Wojczak, and J. Pelzl, J. Nucl. Mater. 162-164 (1989), 935-940.

8. E.R. Müller, B.K. Bein, and K. Steinmetz, Report IPP III/97, Max-Planck-Institut für Plasmaphysik, Garching (1984).

9. B.K. Bein, U. Krebs, and J. Pelzl, High Temp.-High Pressures 21 (1989), 355-361.

10. B.K. Bein, S. Krüger, and J. Pelzl, J. Nucl. Mater. 145-147 (1987), 458-462.

11. B.K. Bein, In: Photoacoustic and Photothermal Phenomena, (Eds. P. Hess and J. Pelzl), Springer Series in Optical Sciences, Vol.58, Springer Berlin, Heidelberg, 1988, 308-311.

12. W. Jabob, D. Boutard, V. Dose, A. Koch, W. Möller, W. Renz, R. Wilhelm, In: Proc. 9th ISPC, Pugnochiuso, Italy, 1989, (Ed. R. d'Agostino, University of Bari), Vol. 3, 1826-1831.

13. B.K. Bein, S. Müller, W. Rubelowski, H.W. Schmidt, B. Wunderlich, J. Pelzl, In: Proc. 10th Intern. Symp. on Plasma Chemistry, Bochum, 1991 (Eds. U. Ehlemann, H.G. Lergon, K. Wiesemann), Vol.4, paper 2.5-7. 